\title{
A influência do "modelo centrado na doença" no uso de medicamentos para problemas de aprendizagem na escola
}

Fabiola Stolf Brzozowski

\section{Resumo}

Nosso objetivo é refletir como o "modelo centrado na doença” para o efeito dos fármacos em psiquiatria, elaborado por Joanna Moncrieff, pode influenciar o uso de medicamentos. Destacamos o caso do metilfenidato (Ritalina ${ }^{\varpi}$ ), utilizado no tratamento do Transtorno de Déficit de Atenção com Hiperatividade (TDAH), identificado a partir de problemas de comportamento ou aprendizagem na escola. Esse diagnóstico costuma ser explicado como resultado de um desequilíbrio cerebral, e o metilfenidato seria um tratamento específico para corrigir esse desequilíbrio, caracterizando o "modelo centrado na doença” para a ação de fármacos no organismo. Argumentamos que esse modelo amplia o processo de medicalização da infância e da aprendizagem escolar, tornando banal a utilização de medicamentos para "corrigir" comportamentos e melhorar o desempenho acadêmico.

Palavras-chave: Medicalização da infância. Metilfenidato. Escola. Modelo centrado na doença.

\section{Introdução}

Definimos medicalização como o processo no qual problemas que não eram considerados de ordem médica passaram a ser vistos e tratados como problemas da área da saúde (ILLICH, 1975; CONRAD; SCHNEIDER, 1992). E isso significa que algumas condiçóes passaram a ser identificadas como doenças ou transtornos que necessitam de cuidados especializados,

I Doutora em Saúde Coletiva, pós-doutoranda no Programa de Pós-Graduação em Ciências Humanas da Universidade Federal de Santa Catarina. O presente trabalho foi realizado com apoio da Coordenação de Aperfeiçoamento de Pessoal de Nível Superior - Brasil (CAPES) - Código de Financiamento 00I. E-mail: fabiola.stolf@ gmail.com.

\section{$(\mathrm{cc})$ Er}

Direito autoral e licença de uso: Este artigo está licenciado sob uma Licença Creative Commons. Com essa licença você pode compartilhar, adaptar, para qualquer fim, desde que atribua a autoria da obra, forneça um link para a licença, e indicar se foram feitas alterações. 
bem como de tratamentos adequados. Como exemplos, podemos citar a medicalizaçáo da sexualidade, do nascimento, do envelhecimento, do sofrimento, da loucura, dentre outros.

Nesse universo, a infância também é alvo de intervençôes médicas. Muitos diagnósticos realizados nessa fase da vida iniciam com demandas escolares. As dificuldades na escola com frequência recebem diagnósticos, com destaque para o Transtorno de Déficit de Atenção com Hiperatividade (TDAH), o Transtorno Desafiante Opositor (TDO), os transtornos de ansiedade etc. (SIGNOR; BERBERIAN; SANTANA, 2017).

As queixas escolares para os profissionais de saúde geralmente se relacionam com comportamentos, falta de atençáo e problemas de aprendizagem. Signor, Berberian e Santana (2017) citam expressóes que os professores costumam utilizar para descrever os estudantes encaminhados: "não conseguem permanecer sentados por muito tempo", "não se engajam nas atividades", "têm dificuldades para aprender", "não conseguem ler", "não aceitam regras", dentre outras. Como resultado, muitos desses encaminhamentos geram prescriçôes de medicamentos.

O principal tratamento do TDAH, foco desse texto, é composto por medicamentos estimulantes do Sistema Nervoso Central (SNC), que inclui as anfetaminas e o metilfenidato. Os produtos à base de metilfenidato comercializados no Brasil são a Ritalina, a Ritalina LA e o Concertå. É considerado o tratamento de primeira escolha e o mais utilizado em casos de TDAH desde o início de sua comercializaçáo, no final dos anos de 1950.

No Reino Unido e nos Estados Unidos, assim como é tendência no mundo inteiro, têm havido um aumento exponencial no número de jovens diagnosticados com TDAH, o que pode ser visualizado a partir do número de pessoas recebendo medicamentos para o transtorno: um aumento de 0,5 usuários para cada 1.000 pessoas $(0,5 / 1.000)$ para $3 / 1.000$ nos últimos 30 anos do Reino Unido e de 12/1.000 para 35/1.000 no mesmo período nos Estados Unidos (SINGH et al., 2010).

As explicaçôes biológicas são muito utilizadas para justificar e legitimar as intervençôes diagnósticas e terapêuticas em crianças com problemas escolares. A biologização, ou seja, a explicação de comportamentos por 
meio da biologia, permite sustentar a ideia de que o TDAH é um transtorno cerebral, tratável com medicamentos que permitiriam corrigir um desequilíbrio ou uma falha do órgão (BRZOZOWSKI; CAPONI, 2015; CAPONI, 2019). Esse tipo de explicação é característica do que Joanna Moncrieff (2008) chama de "modelo centrado na doença", no qual um medicamento é descrito como sendo responsável por corrigir um defeito cerebral. Nesse contexto, nosso objetivo é refletir como o "modelo centrado na doença”, elaborado por Moncrieff, pode influenciar o uso de medicamentos, em especial o metilfenidato para o tratamento de TDAH em crianças com problemas na escola.

Para tal, primeiramente iremos discutir e conceituar os processos de medicalização e biologização da infância. Em seguida, focaremos no tratamento medicamentoso e como o seu uso se insere no cotidiano escolar, apresentando os modelos "centrado na doença" e "centrado no fármaco" descritos por Moncrieff. Por fim, levantamos alguns questionamentos sobre a predominância das explicaçôes centradas na doença e suas consequências para a criança e a escola.

\section{Medicalização da infância e o diagnóstico do TDAH}

Os diagnósticos psiquiátricos incluem várias condiçôes situadas na fronteira entre o normal e o anormal, tais como: a ansiedade, a depressão leve e o TDAH. Mesmo que as classificações psiquiátricas tenham se originado nos manicômios, elas não ficam mais restritas a esses locais. A escola identifica muitos comportamentos que para os manuais diagnósticos, por exemplo, o Manual Diagnóstico e Estatístico de Transtornos Mentais (DSM), publicado pela Associação Americana de Psiquiatria (APA), são considerados sintomas.

Segundo APA (2014) a 5a edição do DSM (DSM-5), o TDAH é considerado um transtorno do neurodesenvolvimento, caracterizado por desatenção, desorganização, hiperatividade e/ou impulsividade. Esse manual descreve a desatenção e a desorganização como "incapacidade de permanecer em uma tarefa, aparência de não ouvir e perda de materiais em níveis inconsistentes com a idade ou o nível de desenvolvimento"; e a hiperatividade e impulsividade como "[...] atividade excessiva, inquietação, 
incapacidade de permanecer sentado, intromissão em atividades de outros e incapacidade de aguardar" (APA, 2014, p. 32). Um dos prejuízos descritos no DSM como consequência do TDAH relaciona-se com limitaçóes no sucesso acadêmico, ou seja, problemas na aprendizagem.

Consideramos o discurso em torno do TDAH como fazendo parte do processo de medicalização, na medida em que encara crianças e adultos com desvios de comportamento (como falta de atenção e agitação) como sujeitos com um transtorno mental. Essa postura ganhou força e legitimidade a partir da incorporação de explicaçóes biológicas cerebrais para os comportamentos considerados patológicos e a partir da possibilidade de tratamento psicotrópico. Muitos aspectos sociais e psicológicos, pelas explicaçóes encontradas atualmente sobre o TDAH, são reduzidos ou até mesmo negligenciados (BRZOZOWSKI; CAPONI, 2014).

A infância nem sempre foi considerada um período da vida que exige cuidados diferenciados ou alvo de preocupação do mundo adulto. Essa mudança foi ocorrendo gradualmente a partir do século XIII. Nesse processo, hábitos e condutas infantis passaram a ser alvos de intervenção. Não era mais recomendado que crianças se beijassem, se tocassem com as mãos ou se olhassem durante brincadeiras, e que dormissem na mesma cama com pessoas mais velhas (o compartilhamento do leito era comum na época). As crianças passaram a ser consideradas criaturas frágeis que deveriam ser preservadas e disciplinadas, sentimento que passou também para a vida familiar. No século XVIII, foram acrescentadas ainda as preocupaçóes com a saúde física e a higiene das crianças e das famílias (ARIĖS, 1981).

Assim, a infância passou a ser vista como um período especial, que necessita de preparo e cuidados diferenciados para a produçáo de indivíduos saudáveis para o trabalho. Como resultado, tiveram início nessa época as intervençôes em escolas e nas famílias, baseadas em teorias higienistas, degeneracionistas, nas concepçóes da puericultura e da psicologia do desenvolvimento (GUARIDO, 2010).

A biologização do sofrimento e do comportamento infantil, e sua transformação em diagnósticos psiquiátricos teve impulso com o nascimento de uma nova psiquiatria a partir da segunda metade do século XX nos Estados Unidos, que se caracteriza por: biologização de transtornos 
metais, vinculada às descobertas psicofarmacológicas; desenvolvimento do campo neurocientífico e de suas tecnologias de imageamento cerebral; crescimento de pesquisas epidemiológicas populacionais baseadas em riscos individuais; desenvolvimento de tecnologias genéticas, biofísicas e bioquímicas (CALIMAN, 2009).

Desde que a categoria "hiperatividade", considerada precursora do TDAH, passou a fazer parte do rol de diagnósticos psiquiátricos da infância, ocorreram várias alteraçóes na classificação inicial, revelando a elasticidade das categorias médicas relacionadas a comportamentos. Nesse caso, primeiramente o diagnóstico era realizado em crianças muito ativas e impulsivas, essencialmente em meninos. A partir da década de 1980, a hiperatividade foi dividindo espaço com a falta de atenção. $\mathrm{O}$ tratamento farmacológico continuou o mesmo (fármacos estimulantes do SNC); porém, a categoria diagnóstica se expandiu, englobando um número maior de pessoas, tais como meninas, adolescentes e adultos (CONRAD; SCHNEIDER, 1992; BRZOZOWSKI; CAPONI, 2014).

No caso dos adultos, o DSM-5 afirma que os sintomas devem estar presentes antes dos 12 anos de idade, o que significa que os comportamentos infantis possuem grande peso para a realizaçáo do diagnóstico. Por essa razão, é muito comum que o TDAH seja identificado com mais frequência durante o ensino fundamental. $\mathrm{O}$ manual também descreve ser frequente que os sintomas de hiperatividade se tornem menos habituais na adolescência e na vida adulta; entretanto, frequentemente persistem a desatenção e a impulsividade (APA, 2014).

O diagnóstico de TDAH, atualmente, é realizado em 2,5\% dos adultos (APA, 2014) e entre 5\% (APA, 2014) e 7,2\% das crianças em idade escolar (THOMAS et al., 2015). No Brasil, estudos apresentam prevalência de aproximadamente 8,6\% (PASTURA; MATTOS; ARAÚJO, 2007). Como consequência, o consumo de psicofármacos, em especial o metilfenidato, alcança valores consideráveis, que serão discutidos mais detalhadamente da seção 3 .

Os primeiros escritos relacionados à falta de atenção em crianças datam do ano de 1798, realizados pelo médico Alexander Crichton. Para ele, essa característica era consequência de um problema nervoso que poderia 
nascer com o indivíduo ou ser o efeito de uma doença posterior ao nascimento. Crichton defendia que, caso fosse um problema de nascença, a falta de atenção seria evidente desde cedo, gerando um resultado muito ruim e tornando a criança incapaz de prender sua atenção por muito tempo em um objeto. Mesmo assim, o autor acreditava que essas crianças eram capazes de aprender e que a desatenção tendia a diminuir com o passar do tempo (FITZGERALD; BELLGROVE; GILL, 2007).

$\mathrm{Na}$ segunda metade do século XIX, foram publicados vários textos sobre "doenças psíquicas", "doenças mentais" ou "insanidade" em crianças. Desvios de comportamento eram descritos por médicos da época e diagnosticados de acordo com as classificaçóes utilizadas para os indivíduos adultos (KANNER, 1959). A loucura antes da puberdade era considerada rara, pois se acreditava que a mente da criança era instável demais para produzir mais do que efeitos transitórios (WALK, 1964). As doenças relatadas geralmente eram reconhecidas como resultados irreversíveis da hereditariedade, degeneração, masturbação excessiva, trabalho excessivo ou preocupação religiosa. Além disso, no início do século XX, depois que a frequência escolar se tornou obrigatória em vários países, os educadores se tornaram mais preocupados com problemas de aprendizagem e de conduta entre os seus alunos (KANNER, 1959), problemas que foram se tornando objetos da área da psiquiatria.

A partir de então, o diagnóstico que hoje conhecemos como TDAH já foi descrito e chamado uma grande variedade de nomes, tais como encefalite letárgica (sequelas), dano cerebral mínimo, disfunção cerebral mínima, hipercinesia e transtorno de déficit de atenção (TDA). Alguns autores defendem que essas doenças descrevem um conjunto de sintomas muito parecidos entre si, caracterizado por vários desvios da infância. Dentre eles, podemos destacar o baixo desempenho escolar, a extroversão extrema, o comportamento violento, a incapacidade de completar tarefas, a ladroagem, os distúrbios nos padróes de sono, a moralidade inconsistente com a idade, o esquecimento (RAFALOVICH, 2001), a falta de atenção, dentre outros.

Enquanto alguns autores localizam em Crichton o início do interesse em crianças que apresentavam baixa capacidade de atenção e hiperatividade, encaixando essas características em um quadro nosológico, outros 
acreditam que esse interesse é mais recente, datando do início do século XX. Em 1901, George Frederick Still ([1901] 2006) relatou uma série de 20 casos de crianças com problemas de hiperatividade, falta de atenção e dificuldades na inibição de suas vontades, como o próprio autor descrevia. Segundo Rafalovitch (2001), Still foi o primeiro a ligar a noção de debilidade mental à moralidade de crianças, embora ele não tenha fornecido um diagnóstico oficial para estes comportamentos.

Para Timimi (2002), foi somente alguns anos mais tarde que os sintomas de hiperatividade e falta de atenção foram relacionados e considerados como fazendo parte da mesma patologia. De acordo com o autor, essa relação se deu com o surgimento do diagnóstico de "dano cerebral mínimo", após as epidemias de encefalite. A encefalite letárgica, também conhecida como doença do sono (sleepy sickness) é uma forma atípica de encefalite, cujas causas ainda não são totalmente conhecidas. Essa doença alcançou proporçôes epidêmicas entre 1916 e 1927 (DALE et al., 2004), principalmente na América do Norte e na Europa, levando grande parte das pessoas acometidas à morte.

Por se originar de uma doença residual, cuja causa acreditava-se ser infecciosa (na época, pensava-se que a encefalite estava relacionada com a epidemia de influenza), os sintomas "pós-encefalíticos" foram considerados fisiológicos e passíveis de localização cerebral. Dessa forma, comportamentos como irritabilidade, agitação e problemas de atenção puderam ser explicados por vias biológicas, abrindo caminhos para as pesquisas com esse tipo de viés.

A descoberta da ação de psicoestimulantes, na década de 1930, para a redução da inquietação, da hiperatividade e dos problemas de comportamento em crianças forneceu mais subsídios para as hipóteses de que lesóes orgânicas no cérebro seriam responsáveis pela hiperatividade e falta de atenção em outras crianças que não haviam apresentado encefalite, levando à criação do diagnóstico de "dano cerebral mínimo" (TIMIMI, 2002). O termo "psiquiatria infantil" também foi introduzido nessa época, sendo legitimado em 1937 no primeiro congresso internacional de psiquiatria infantil, em Paris (KANNER, 1959).

Com o passar dos anos, não foram encontradas lesóes cerebrais orgânicas em crianças mais agitadas e o termo "dano cerebral mínimo" foi sendo 
substituído por "disfunção cerebral mínima" a partir da década de 1960. No DSM-I (APA, 1952), publicado em 1952, não aparece um diagnóstico de crianças pós-encefalite, nem outro que possa se considerar equivalente ao TDAH hoje, com sintomas como falta de atenção ou agitação. Os estudos com crianças não utilizavam o DSM como base para as nomenclaturas de transtornos mentais (BRZOZOWSKI; CAPONI, 2014).

Somente na segunda ediçấo do DSM, publicada em 1968 (APA, 1968), surgiu um diagnóstico para falta de atenção e agitação em crianças, e a "disfunção cerebral mínima" passou a ser chamada de "reação hipercinética da infância" (TIMIMI, 2002). Entretanto, o DSM ainda não era considerado um manual padrão para diagnóstico, coexistindo várias nomenclaturas em estudos publicados na época. Essa variabilidade de diagnósticos poderia ser um indício também da variabilidade de situaçóes que são classificadas atualmente como TDAH.

O quadro mudou com a publicação do DSM-III, em 1980 (APA, 1980). Em relação aos sintomas da "reação hipercinética da infância", foram enfatizados os aspectos atencionais como determinantes para o diagnóstico, alterando a nomenclatura para "Transtorno do Déficit de Atenção" (TDA). O DSM-III foi considerado um marco de uma grande mudança na psiquiatria, inaugurando uma nova forma de abordagem dos transtornos mentais, com o objetivo de uniformizar os diagnósticos. Passou a apresentar listas de sinais e sintomas diferentes para cada classificação, numa tentativa de se aproximar de outras áreas da medicina através da objetividade (MARTINHAGO; CAPONI, 2019).

O DSM-IV (APA, 1994), na década de 1990, dividiu a classificaçáo do TDA em três subtipos: um tipo predominantemente desatento, um tipo predominantemente hiperativo e/ou impulsivo, e um tipo combinado. Nessa versão do manual, o transtorno passou a se chamar Transtorno de Déficit de Atençáo com Hiperatividade, ou TDAH. Vale ressaltar que a mudança do DSM-III para o DSM-IV, com critérios mais abrangentes, aumentou a prevalência do transtorno em aproximadamente dois terços, uma vez que os novos critérios passaram a englobar grande parte das crianças com problemas de aprendizagem e comportamento na escola (TIMIMI, 2002).

O DSM-5 mantém os três subtipos do manual anterior, sendo criticado por ser ainda mais abrangente. De acordo com Allen Frances (2012), 
os diagnósticos da quinta edição do manual foram incluídos por necessidade prática, por acidente histórico, por precedente e inércia, mas não por serem critérios universais. Além disso, apresenta um número ainda maior de diagnósticos, quando comparado com o DSM-IV, com destaque para as patologias relacionadas à prevenção e à antecipação de riscos, por exemplo, a identificação de sinais preditores de transtornos em crianças (MARTINHAGO; CAPONI, 2019). O discurso do risco na psiquiatria não é novo, e está presente já no início da década de 1980, com a preocupação de que a criança hipercinética poderia desenvolver transtornos psiquiátricos quando chegasse à vida adulta, ou poderia se tornar um delinquente ou usuário de drogas (BRZOZOWSKI; CAPONI, 2014).

A incorporação de diagnósticos psiquiátricos no cotidiano escolar, principalmente a partir do século XX, se baseia nas explicaçóes de cunho organicista e biológico para as dificuldades de aprendizagem e para os problemas de comportamento na escola (SIGNOR; BERBERIAN; SANTANA, 2017). Ao encarar comportamentos como biologicamente determinados, é razoável considerar medicamentos como correção daqueles que porventura forem considerados inadequados. Abordaremos essa temática com mais detalhes na seção 3 .

\section{Inserção do metilfenidato no cotidiano escolar}

Ao mesmo tempo em que a noção de infância foi surgindo, a instituição escolar também foi mudando, assumindo um papel de controle dos comportamentos das crianças. A partir do século XV, os chamados colégios passaram a ser centros de ensino. A instituição de regras rígidas de disciplina marcou a evolução entre a escola medieval (uma simples sala de aula) para o colégio moderno (ARIÈS, 1981). A partir da metade do século XIX e início do século XX, a saúde e a higiene passaram a fazer parte das preocupaçóes da escola e a instituição começou a participar ativamente de campanhas que visam ao combate de endemias e epidemias, como as de vacinação e de prevenção em saúde (ROCHA, 2003). Os discursos higienistas na escola permitiram a entrada da área da saúde na instituição, abrindo espaço para a identificação e o encaminhamento ao médico de crianças com problemas de comportamento. 
Segundo Conrad e Schneider (1992), escolares mais agitados e que não prestam muita atenção sempre foram considerados um problema para pais e professores. Mas a ideia de que esses comportamentos sáo sintomas de um transtorno mental, tratáveis com medicamentos, é mais recente. Atualmente, a escola pode ser também local de identificaçâo de sinais de riscos para o desenvolvimento de transtornos mentais na idade adulta. Identificando as crianças em risco, seria possível atuar enquanto são pequenas e evitar que se tornem adultos com problemas (LIMA; CAPONI, 2011). No Brasil, o Instituto Nacional de Psiquiatria do Desenvolvimento para Crianças e Adolescentes (INPD), criado em 2009, é o grande difusor dessas ideias no país. Defende que os transtornos mentais estáo relacionados com o desenvolvimento cerebral e que podem ser revertidos através de intervençóes precoces, até mesmo no período intrauterino.

Alguns autores argumentam que a medicalizaçáo da educaçáo e a consequente biologização dos comportamentos vem sendo utilizada para justificar o fracasso escolar. Signor, Berberian e Santana (2017) afirmam que esse tipo de atitude oculta condicionantes sociais, culturais, políticos, afetivos e educacionais que podem estar relacionados com as condutas das crianças na escola. Como dito anteriormente, uma das consequências desse processo de medicalizaçáo é o consumo de medicamentos.

Um medicamento bastante comum no cotidiano escolar é o metilfenidato, sintetizado em 1944 e, inicialmente, utilizado por adultos para o tratamento de quadros depressivos, como tônico para velhice e no tratamento de cansaço. Depois dos anos de 1960 que o metilfenidato passou a ser mais frequentemente utilizado em crianças com problemas escolares. Na década de 1990, um estudo clínico randomizado, o MTA (Multimodal Treatment Study), que durou 14 meses, avaliou a eficácia e a tolerância do metilfenidato em 579 crianças com TDAH, sugerindo benefícios desse medicamento (MTA, 1999). Essa pesquisa foi considerada um marco e os guidelines de psiquiatria passaram a descrever os estimulantes como o tratamento de primeira escolha para o TDAH (FINDLING, 2008).

As explicaçôes que predominavam até a década de 1970, para os problemas de aprendizagem e de comportamento na escola, eram de cunho social. Apesar disso, as pesquisas da época sobre estimulantes descreviam 
esses medicamentos como apresentando efeitos favoráveis em crianças pós-encefalíticas e hipercinéticas, produzindo um comportamento submisso, aumentando o tempo de concentração, resultando no incremento do desempenho escolar (BRZOZOWSKI; CAPONI, 2015).

Nas duas últimas décadas, o uso de medicações para TDAH em jovens apresentou elevação significativa (BACHMANN et al., 2017). O número de países importadores de metilfenidato aumentou em 2018, enquanto o seu consumo teve um pequeno declínio em muitos países, conforme cita International Narcotics Control Board (INCB, 2020). Apesar de esses dados darem a impressão de redução no consumo, não é o que ocorre: os estimulantes em geral continuam sendo os tratamentos mais frequentes para o TDAH (SINGH, 2013). Apenas no Brasil, entre 2003 e 2012, ocorreu um aumento de $775 \%$ na utilização de metilfenidato (BARROS, 2014)

A prevalência de uso de medicamentos para TDAH entre crianças e jovens é de 3,9\% nos Países Baixos, 3,7\% nos Estados Unidos, 2,2\% na Alemanha, $1,5 \%$ na Dinamarca e $0,5 \%$ no Reino Unido. $\mathrm{O}$ uso desses medicamentos é maior na faixa etária de 10 a 14 anos. O consumo de metilfenidato predomina na Europa, enquanto nos Estados Unidos é comum a utilização de anfetaminas (BACHMANN et al., 2017).

Esses números são elevados, trazendo preocupaçóes sobre o uso excessivo de estimulantes e seus efeitos adversos em longo prazo em pessoas mais jovens (SINGH et al., 2010). Crianças e adolescentes usam esses medicamentos, mas seus pais afirmam com frequência que utilizam esses produtos exclusivamente no período escolar, justificando pausas nas medicaçôes durante finais de semana e férias (SINGH, 2013). No estudo de Singh (2013, p. 364), uma das crianças afirmou: "[no verão eu não tomo o medicamento] porque posso só relaxar lá, porque eu ando de bicicleta todos os dias. Estou sempre ativa, o que eu quero fazer. Portanto, estou bem no verão, mas não no período escolar".

Essa fala ilustra que os comportamentos do TDAH parecem ser um problema no ambiente escolar. As formas de avaliar esses comportamentos, e se um tratamento resulta em melhora ou não, são as escalas, tais como o Teacher's Report Form (TRF) e o The Strengths and Difficulties Questionnaire (SDQ), questionários preenchidos por professores que têm como objetivo identificar problemas de comportamento em crianças e adolescentes em 
idade escolar. Alguns dos quesitos avaliados são agressividade, questôes sociais, ansiedade, problemas de atenção, dentre outros.

Além dos comportamentos, estudos científicos descrevem a melhoria do desempenho acadêmico como um dos alvos do tratamento com estimulantes em crianças com TDAH. Um artigo de 2019 sobre a produtividade acadêmica em crianças com déficit de atenção, de Kortekaas-Rijlaarsdam et al. (2019), intitulado "Does methylphenidate improve academic performance? A sistematic review and meta-analyses", destaca a melhoria nessa qualidade como um dos objetivos da terapêutica. Os autores descreveram um aumento de 3\% na precisão matemática e 7,8\% na produtividade dessa matéria. A acurácia na leitura, por sua vez, não sofreu alteraçóes. Os autores ressaltam que todos os estudos analisados eram de curto prazo. O texto afirma, ainda, que "[...] melhoras no comportamento na sala de aula são geralmente encaradas como um pré-requisito para melhoras acadêmicas” (KORTEKAAS-RIJLAARSDAM et al., 2019, p. 162). E há uma crença de que "[...] continua sendo possível que os efeitos de curto prazo do metilfenidato no desempenho, tanto comportamental quanto acadêmico (isto é, produtividade), [...] possam se traduzir em benefícios de longo prazo" (KORTEKAAS-RIJLAARSDAM et al., 2019, p. 162).

Muitos estudos relacionados ao melhoramento acadêmico são inconclusivos e focam no engajamento de tarefas. Os resultados considerados de melhoria costumam estar relacionados mais com produtividade do que com acurácia. Algumas características comuns que os estudos utilizam para medir desempenho acadêmico são produtividade e acurácia em matemática, leitura e soletração.

Parece que, de forma geral, a literatura científica destaca o efeito positivo do metilfenidato em crianças e adolescentes com TDAH, em relação ao desempenho na escola. Entretanto, a efetividade em longo prazo não é clara (VAN DER SCHANS et al., 2017). Por exemplo, uma revisão sistemática de 2015 afirma que o metilfenidato apresenta efeitos positivos em crianças com TDAH, segundo os seus professores. Ao mesmo tempo, tal revisão destaca a baixa qualidade das pesquisas incluídas, questionando a "eficácia real" do tratamento avaliado (STOREB $\varnothing$ et al., 2015).

Nesse sentido, Van der Schans et al. (2017) afirmam que os usuários de metilfenidato apresentam desempenho escolar menor do que crianças 
sem história de uso de medicamentos para TDAH. Além disso, o início precoce do tratamento resultou em menor desempenho escolar. Segundo os autores, isso indica que o metilfenidato não normaliza o desempenho e que o tratamento em longo prazo parece ser menos efetivo.

Apesar dos dados duvidosos, a literatura da área de psiquiatria e neurologia sobre a ação do metilfenidato em pessoas com TDAH assumem a ideia de uma normalização cerebral: "Estimulantes parecem melhorar os sintomas em jovens com TDAH por normalizar as atividades nesse circuito [córtex cingulado anterior ventral e cingulado posterior] e melhorar suas interaçóes funcionais com o córtex pré-frontal lateral" (PETERSON et al., 2009 , p. 1286). A normalização biológica é a premissa básica que sustenta e legitima a grande maioria dos estudos sobre o metilfenidato atualmente (BRZOZOWSKI; CAPONI, 2015). Admite-se, nesse contexto, que a normalização dos circuitos cerebrais resultaria numa normalização de suas funções e dos comportamentos individuais.

Ao ouvir crianças e adolescentes que utilizam medicamentos para tratar o TDAH, Singh (2013) observou que eles encaram a medicação como uma ferramenta que permite que cumpram com suas obrigaçóes, incluindo terem sucesso em suas atividades cognitivas (SINGH, 2013). Singh et al. (2010) citam como efeitos positivos dos medicamentos o fato de deixarem crianças e adolescentes mais calmos, conseguindo pensar antes de agir e de não se sentindo irritados; além disso, afirmam que esses efeitos possuem impacto na habilidade de fazer e manter amizades.

Uma parte dos entrevistados de Singh et al. (2010) afirmou que a medicação teve um efeito positivo em sua concentração. Cerca de metade descreveu incrementos nas tarefas escolares, em escrita e matemática, e nas notas com o uso de medicamentos. Outros relataram que a medicaçáo melhorou o foco e a concentração, mas que continuaram com problemas nos trabalhos escolares.

A utilização de medicamentos para tratar problemas de comportamento e aprendizagem na escola foi sendo naturalizada ao longo do tempo, mesmo que esses produtos apresentem efeitos negativos. Alguns dos efeitos do metilfenidato são: perda de apetite, insônia, irritabilidade, cefaleia, sintomas gastrintestinais, interferência no crescimento, potencial de 
abuso, sintomas psicóticos e morte súbita (GOULD et al., 2009; LEE et al., 2011; ROSS, 2006). Não utilizar medicamentos prescritos pode ser até mesmo interpretado como um desinteresse ou descaso. Isso ocorre porque situaçôes são consideradas "patológicas" de acordo com o que se considera normal em determinadas épocas e sociedades. $\mathrm{O}$ uso de estimulantes em crianças passa a ter um viés moralista, cuja crença é de que a criança somente consegue controlar seu comportamento com esse uso (BRZOZOWSKI; CAPONI, 2015).

As explicaçóes de cunho biológico e cerebral para problemas de comportamento e utilização de medicamentos sustentam o processo de medicalização da infância e de moralização de certas condutas. Os medicamentos, em especial, ao serem descritos como específicos para o tratamento de determinadas situaçôes, dão a impressão de que são capazes de "reequilibrar" o cérebro, cujo desequilíbrio seria responsável pelo aparecimento da doença/transtorno. Joanna Moncrieff $(2008,2013)$ ajuda a entender esse tipo de explicaçóes, através de dois modelos para o entendimento de como os psicofármacos agem nos organismos: um modelo centrado na doença e um modelo centrado no fármaco. Vamos apresentar primeiramente cada um deles, de acordo com os conceitos de Moncrieff para, na seção 4, abordar as consequências da utilizaçáo de cada um deles no caso do metilfenidato.

A afirmação de que os tratamentos medicamentosos psiquiátricos modernos são específicos para determinados transtornos é o que Moncrieff (2008) chama de "modelo centrado na doença". Nesse caso, diz-se que os fármacos agiriam em processos biológicos subjacentes dos transtornos mentais, revertendo aqueles que são anormais. Não se fala necessariamente em uma cura, mas em alvos específicos, responsáveis pelos sintomas. Por exemplo, os antipsicóticos reestabeleceriam um desequilíbrio da dopamina, responsável pelos sintomas psicóticos agudos, sem curar a causa desse desequilíbrio.

Esse modelo, segundo Moncrieff (2008), é importado da medicina geral, na qual a maioria das açóes dos fármacos pode ser entendida dessa forma. Por exemplo, a reposição da insulina para indivíduos com diabetes tipo I e os fármacos antianginosos fazem com que o corpo que náo produz insulina e/ou aquele que apresenta obstrução momentânea das coronárias cardíacas possa voltar a funcionar normalmente. Esses e outros medicamentos agem em processos biológicos que causam os sintomas das doenças 
que são alvo dos tratamentos. Em alguns casos, pode-se falar que os medicamentos curam a doença, como é o caso dos antibióticos para infecçóes bacterianas, mas uma parte deles simplesmente ameniza os sintomas, como os exemplos citados.

No modelo centrado na doença, a ação básica de um fármaco pode ser dividida em efeitos terapêuticos e efeitos colaterais. Os efeitos terapêuticos são os desejados, relacionados ao processo patológico, e os colaterais são os efeitos diferentes dos desejados. Por exemplo, a morte das células tumorais nos tratamentos quimioterápicos convencionais gera seus efeitos terapêuticos; porém, a morte de outras células do corpo que se replicam rapidamente (como as dos folículos capilares, da medula óssea ou da mucosa bucal) é um efeito colateral.

No caso da psiquiatria, num modelo centrado na doença, os antipsicóticos agiriam especificamente nas causas das psicoses, os antidepressivos nas causas da depressão, os ansiolíticos nas bases patológicas da ansiedade, os medicamentos para TDAH nas causas da agitação e falta de atenção, e assim por diante. Apesar de os estimulantes não serem chamados de "anti-TDAH”, como nos casos dos antidepressivos, antipsicóticos etc., as explicaçóes dadas para seus efeitos sugerem que tenham uma ação de correção sobre uma causa cerebral subjacente.

O modelo centrado na doença, portanto, sugere que os efeitos terapêuticos dos fármacos ocorram por seus efeitos em um processo patológico específico, fazendo com que o organismo retorne para uma condição biológica de normalidade. $\mathrm{O}$ modelo centrado no fármaco, proposto por Moncrieff (2008), por sua vez, sugere que os próprios fármacos criam um estado anormal no organismo. No caso dos psicofármacos, esse estado envolve uma alteraçáo na experiência subjetiva ou na consciência.

A autora afirma que é fácil notar esse tipo de explicação no uso de drogas recreativas: elas alteram o funcionamento cerebral normal, gerando os seus efeitos. Moncrieff (2008) argumenta que isso ocorre também no caso de psicofármacos: sua administração não corrige uma anormalidade biológica, mas gera um novo estado cerebral. As ações desses fármacos variam de acordo com a natureza química, sua interação com o cérebro e as variabilidades individuais momentâneas (contexto de administração, questóes sociais e emocionais etc.). 


\section{O modelo centrado no fármaco sugere que}

[...] o valor terapêutico de um fármaco é derivado de uma qualidade particular de um estado anormal que ele produz. Alguns efeitos induzidos por fármacos podem ser úteis ou desejáveis em certas situações sociais e interpessoais, incluindo situações que despertam a atenção dos psiquiatras e são chamadas de transtornos mentais. (MONCRIEFF, 2008, p. 15).

Moncrieff (2008) cita o exemplo do álcool para explicar o potencial terapêutico de uma substância, baseada no modelo centrado no fármaco. O álcool é uma substância sedativa que reduz a condutividade nervosa no sistema nervoso central. Alguns de seus efeitos sáo dilatação de vasos sanguíneos, relaxamento muscular e redução do tempo de reação, que produzem alteraçôes nos comportamentos e na experiência subjetiva. Em baixas doses leva à euforia, à desinibição social e a danos da função intelectual, podendo ser até considerado terapêutico em certas circunstâncias.

O modelo centrado no fármaco para a ação dos psicofármacos prescritos sugere que se pode entender seus efeitos da mesma forma que entendemos os efeitos de drogas recreativas. Uma evidência disso é o fato de que os fármacos psiquiátricos podem ser também drogas de abuso. Assim, levando em consideraçáo o modelo centrado na doença e o centrado no fármaco, na seção 4 iremos abordar como esses dois modelos se aplicam para o metilfenidato e as consequências para a escola e para a vida dos estudantes.

\section{0 modelo centrado na doença e uso de metilfenidato para problemas na escola}

A utilizaçáo de argumentos com base em discursos biológicos, procurando explicaçóes no estilo do modelo centrado na doença, reforça a ideia de que é indispensável utilizar medicamentos para tratar transtornos mentais, além de dar esperanças de que em breve haverá substâncias para melhorar capacidades cognitivas, para consolar as tristezas, controlar as ansiedades etc. Esses controles sáo essenciais para o mundo neoliberal, que requer um novo modo de exercer o poder, por meio da "[...] implantação geral de uma nova lógica normativa, capaz de incorporar e reorientar duradouramente políticas e comportamentos numa nova direçâo" (DARDOT; LAVAL, 2016, p. 190). 
O metilfenidato foi inicialmente aprovado e comercializado para o tratamento de situaçóes específicas, dentre elas o TDAH, caracterizado por sintomas como falta de atenção e hiperatividade. Com as revisôes dos critérios diagnósticos para o TDAH e as suas expansóes, cada vez mais crianças e, posteriormente, adultos, foram sendo incorporados a essa categoria. Como consequência, ocorreu também a expansão no uso de estimulantes, em especial o metilfenidato. O possível mecanismo de ação desses medicamentos, relacionado com neurotransmissores cerebrais, em especial a dopamina, e sua resposta frente à falta de atenção e à hiperatividade, tornaram plausíveis as explicaçóes biológicas para os comportamentos, conferindo legitimidade à ideia de que o TDAH é uma condição causada por um desequilíbrio neuronal (BRZOZOWSKI; CAPONI, 2015).

Assim como Sandra Caponi (2019) afirma que, no caso da clorpromazina, foi o modelo centrado na doença que permitiu a consolidação, a aceitação e a sustentação do uso desse e de outros psicotrópicos para o tratamento de transtornos mentais, podemos afirmar que isso ocorreu também no caso do metilfenidato. Atualmente, o uso desse medicamento na infância parece ter dois objetivos primários principais: a melhoria do comportamento, relacionada com a disciplina, e a melhoria do desempenho, relacionada com um discurso de produtividade.

$\mathrm{O}$ modelo centrado na doença fornece à psiquiatria um discurso que lhe confere certo poder em relação aos pacientes. Essa pode ser uma das razóes para que esse modelo seja hegemônico. Caponi (2019) descreve os efeitos biopolíticos de controle e disciplina possíveis a partir da adoção de um modelo centrado na doença para explicar os efeitos dos fármacos psiquiátricos: os medicamentos como dispositivos disciplinares para a gestão dos pacientes e a influência da indústria farmacêutica nesse processo.

Caponi argumenta que os neurolépticos possuem um papel político como dispositivo disciplinar para impor o silêncio e a tranquilidade em hospitais psiquiátricos. Da mesma forma, o metilfenidato permite impor a disciplina e a tranquilidade em sala de aula, influenciando no comportamento das crianças. O sucesso do poder disciplinar, segundo Foucault (2008), deve-se a mecanismos eficientes, tais como o olhar hierárquico e a sanção normalizadora. $\mathrm{O}$ primeiro relaciona-se à vigilância e à fiscalização. 
Ressalta-se que, na escola, há vários vigilantes hierárquicos da criança: seus professores, o diretor, o orientador pedagógico, o psicólogo e até o próprio médico. A sanção normalizadora objetiva normalizar os comportamentos anormais, por atividades adicionais para aqueles que não seguem as normas, pelas notas baixas ou ao deixar a criança sem intervalo por algum comportamento etc. Devido a essa percepção, o metilfenidato passou a ser também um dispositivo para manter a disciplina.

Mas além de dispositivo disciplinar, o metilfenidato pode ser também considerado um dispositivo de segurança (FOUCAULT, 2010). Novamente traçando um paralelo com a análise de Caponi (2019) sobre os antipsicóticos atípicos, podemos afirmar que o metilfenidato é utilizado como agente de controle de risco ou de prevenção. O tratamento medicamentoso de "transtornos mentais leves", fora dos hospitais, como é o caso do TDAH, permite a gestão dos pacientes e seu controle, além de uma gestão da segurança, uma vez que as crianças com TDAH são consideradas de risco para delinquência e transtornos mentais mais graves na idade adulta.

Por fim, para a indústria é muito mais rentável e interessante lançar um medicamento que trata uma condição específica, ou seja, cujas explicaçóes estejam baseadas no modelo centrado na doença, do que um tratamento que não necessariamente será em longo prazo. Explicaçóes centradas no fármaco para os efeitos do metilfenidato poderiam fazer com que seu uso fosse mais limitado para determinadas situaçóes e determinados períodos de tempo, não mais para toda a vida, já que não serviriam para corrigir um desequilíbrio perdido. Em outras palavras, ao levar em consideração o modelo centrado na doença, partindo do pressuposto de que uma substância corrige um déficit ou desequilíbrio fisiológico, é lógico pensar que não é possível ficar sem o medicamento, pois somente com este seria possível resgatar a normalidade.

Apesar de ser considerado um tratamento para o TDAH, o metilfenidato apresenta o mesmo efeito tanto em pessoas com esse diagnóstico quanto em pessoas consideradas "normais". Um artigo da década de 1980 já confirmava esse tipo de resposta: “[...] estudos recentes mostraram que tanto crianças normais quanto crianças enuréticas ${ }^{2}$ respondem em muito

2 Crianças com perdas involuntárias de urina durante o sono. 
da mesma maneira como o fazem as crianças hipercinéticas” (RUTTER, 1982, p. 30). E esse é um dos motivos pelos quais o fármaco é muito utilizado para melhorar o desempenho acadêmico. Nesse caso, a biopolítica não se relaciona diretamente à vida e à morte, ao controle da natalidade $\mathrm{e}$ da mortalidade, mas da produtividade.

No mesmo sentido que Foucault (2010, p. 207) analisa os mecanismos para encompridar a vida, o melhoramento cognitivo trata de "[...] estabelecer mecanismos reguladores que [...] vão poder fixar um equilíbrio, manter uma média, estabelecer uma espécie de homeostase, assegurar compensaçóes; [...] de otimizar, se vocês preferirem, um estado de vida".

O uso de substâncias para melhorar alguma característica populacional pode criar uma nova média e cada vez mais pessoas precisarão fazer uso delas para que consigam alcançar essa nova média. E parece ser o que ocorre com o metilfenidato: inicialmente utilizado em crianças com problemas mais graves, logo passou a ser prescrito por crianças agitadas e com dificuldades de concentração na escola, chamadas de TDAH e, por fim, passou a ser usado por pessoas sem diagnóstico.

As exigências para o que se considera inteligência, bom desempenho ou bom comportamento são definidas por especialistas. Foucault (2010, p. 22) afirma que "[...] não há exercício do poder sem uma certa economia dos discursos de verdade que funcionam nesse poder, a partir e através dele". A norma estabelecida por um saber, pelos especialistas de certo campo de conhecimento, constitui a verdade desse campo. Por exemplo, a decisão dos valores de Quociente de Inteligência (QI) de cada idade é realizada por especialistas, podendo até variar ao longo do tempo (FLYNN, 1984). Como descrito na seção 2 , o que já foi considerado comportamento infantil normal e anormal mudou ao longo do tempo. A configuração do TDAH hoje é diferente do diagnóstico de "dano cerebral mínimo", por exemplo. Essas alteraçóes dependem dos conhecimentos acumulados ao longo do tempo, mas também de decisóes políticas, passando pelo que os especialistas de uma sociedade determinam como normal ou não.

O desempenho acadêmico insuficiente e os problemas de comportamento sáo problemas para uma sociedade de produtividade e competitividade. A ênfase na produtividade faz parte de uma reorientaçáo dos comportamentos citada por Dardot e Laval (2016) para a permanência e 
funcionamento de um livre mercado, característica do neoliberalismo. Segundo os autores, "[...] a política neoliberal deve mudar o próprio homem [...] o homem é um inadaptado crônico que deve ser objeto de políticas específicas de readaptação e modernização" (DARDOT; LAVAL, 2016, p. 91).

As mudanças de comportamento citadas por Dardot e Laval (2016, p. 92), necessárias para a existência do neoliberalismo, ficam claras ao analisarmos esse ponto: a criação de uma "[...] grande política de educaçăo das massas que prepare os homens para as funçóes econômicas especializadas que os aguardam e para o espírito do capitalismo a que devem aderir para viver". Para isso, o indivíduo deve ser produtivo, competitivo, empresarial e comportado.

O treinamento para esse novo homem neoliberal começa na escola, com a separação dos inadaptados, dos que não são capazes de darem voz a esse discurso, dos que têm problemas de comportamento ou de aprendizagem. Esses indivíduos são propensos a receberem diagnósticos psiquiátricos. A tentativa de normalizar a criança encontra nas explicaçōes biológicas, em especial no modelo centrado na doença, argumentos científicos praticamente irrefutáveis a favor do uso de medicamentos.

Num mundo de desempenho, os resultados cognitivos das crianças são enfatizados. Em consequência, o TDAH acaba sendo visto como um transtorno de desempenho acadêmico e de comportamento e o tratamento medicamentoso busca corrigir essas duas linhas de ação. Isso acaba sendo táo significativo que as crianças e os adolescentes que fazem uso de metilfenidato acabam sentindo que a medicação é necessária e que é uma parte normal de sua vida (SINGH et al., 2010).

Tratar agitação como um problema médico pode levar a criança a mudar o entendimento de si mesma. Nesse sentido, alguns argumentam que o TDAH é um transtorno válido, e que o medicamento seria capaz de revelar um self mais autêntico, enquanto outros afirmam que os comportamentos característicos do TDAH são parte do verdadeiro self e que a medicaçăo nega o direito a uma vida autêntica (SINGH, 2013).

Singh et al. (2010) chamam a atenção também para o estigma, afirmando que ele está geralmente relacionado com o diagnóstico do TDAH e aos sintomas comportamentais, mais do que com o uso de medicação. Entretanto, os jovens se sentem expostos pela necessidade de tomar seus 
medicamentos, principalmente se precisam fazer isso na escola. Relataram serem chamados de alguns apelidos e sofrerem bullying por seus comportamentos TDAH, pelo diagnóstico e pelo uso de comprimidos. Para os autores, isso tem um impacto no entendimento de si mesmo (self), resultando em baixa autoconfiança e baixa autoestima.

Resgatando os dois modelos formulados por Moncrieff (2008), a visão de que fármacos como o metilfenidato são específicos para o tratamento de determinado transtorno mental, no caso o TDAH, por corrigirem um desequilíbrio neuronal, característica do modelo centrado na doença para a explicação dos efeitos desse medicamento, permite considerar que somente é possível um retorno à normalidade com o seu uso. Esse uso, por sua vez, é um dispositivo para a existência de um poder disciplinar, para a gestão das populaçóes e para o enriquecimento de uma indústria bastante rentável, como já dito anteriormente.

Por outro lado, encarar os efeitos dos psicofármacos a partir de um modelo centrado no fármaco, para o qual essas substâncias não seriam capazes de reestabelecer um desequilíbrio neuroquímico perdido, mas criariam alteraçóes cerebrais que podem ser benéficas para alguns indivíduos em determinadas situaçóes, permitiria que o próprio paciente decidisse a utilidade de um fármaco, de acordo com sua experiência subjetiva e os efeitos particulares em cada um (CAPONI, 2019). Dessa forma, o uso de psicofármacos não seria obrigatório para o retorno a uma "normalidade cerebral" e poderia se dar por um período mais curto de tempo, sendo gerido pelo próprio paciente.

Como consequência individual, especificamente em crianças, o modelo centrado na doença, o diagnóstico de TDAH e o uso de medicamentos para o seu tratamento impactam na construçáo dos próprios sujeitos. Muitas delas admitem usar seus diagnósticos como desculpas para seus comportamentos. As crianças geralmente se sentem responsáveis por eles e afirmam que os medicamentos ajudam com seu autocontrole de alguma forma, ainda que não pensem que a medicação sozinha possa resolver seus problemas (SINGH et al., 2010).

\section{Considerações finais}

A quantidade de pessoas diagnosticadas com TDAH aumentou nos últimos anos e, como consequência, aumentou também a quantidade de 
pessoas medicadas. Dessa forma, substâncias consideradas de uso crônico em saúde mental se tornaram um investimento lucrativo para as indústrias produtoras, primeiramente por essas situaçóes serem consideradas transtornos para a vida toda e, em segundo lugar, por serem condiçốes bastante elásticas, podendo englobar um número cada vez maior de pessoas.

O metilfenidato é um medicamento relativamente comum nas escolas, utilizado por crianças principalmente para o tratamento do TDAH. Sua indicaçáo se dá por dois motivos principais: melhoria do comportamento e melhoria do desempenho acadêmico. As críticas a seu uso relacionam-se ao controle e à transformação de questóes sociais e culturais em problemas biológicos, através da medicalizaçáo da aprendizagem e da indisciplina. Essa medicalização apenas é possível por meio de um discurso biológico subjacente, que afirma que o TDAH é um transtorno cerebral, que pode ser corrigido por medicamentos. Esse discurso é o que Moncrieff (2013) chama de modelo centrado na doença, que permite a existência da gestão do paciente, da cientifização da psiquiatria e da cronificação de uma condição psiquiátrica, tornando esses indivíduos usuários crônicos de medicamentos.

Não se pode negar que os estimulantes tenham ação em crianças com TDAH; entretanto, essa ação ocorre também em pessoas "normais" (prova disso é seu uso para melhorar o desempenho acadêmico antes de provas e concursos). Isso indica que o metilfenidato não é específico para "combater" uma anormalidade biológica, de acordo com o modelo centrado na doença. Se o metilfenidato é um tratamento sintomático, ele pode ser útil para várias condiçóes diferentes, não sendo específico para o TDAH.

O modelo centrado na doença colabora com a crença da necessidade de um tratamento biológico medicamentoso. E isso cai bem num contexto de empreendedorismo e competitividade na escola, no qual a educaçáo de crianças se dá para um mundo individual, no qual cada um é empreendedor de si e deve ser o tempo inteiro produtivo. Essa é uma forma de governo, com o objetivo de tornar as pessoas mais saudáveis, produtivas, empreendedoras, felizes e inteligentes.

\section{Referências}

AMERICAN PSYCHOLOGICAL ASSOCIATION (APA). DSM-I: Diagnostic and Statistical Manual of Mental Disorders. 1. ed. Massachusetts; Washington: APA, 1952. 
A influência do "modelo centrado na doença" no uso de medicamentos para problemas de aprendizagem na escola | Fabíola Stolf Brzozowski

AMERICAN PSYCHOLOGICAL ASSOCIATION (APA). DSM-II: Diagnostic and Statistical Manual of Mental Disorders. 2. ed. Washington: APA, 1968.

AMERICAN PSYCHOLOGICAL ASSOCIATION (APA). DSM-III: Diagnostic and Statistical Manual of Mental Disorders. 3. ed. Washington: APA, 1980.

AMERICAN PSYCHOLOGICAL ASSOCIATION (APA). DSM-IV: Diagnostic and Statistical Manual of Mental Disorders. 4. ed. Washington: APA, 1994.

AMERICAN PSYCHOLOGICAL ASSOCIATION (APA). DSM-5 - Manual Diagnóstico e Estatístico de Transtornos Mentais. Porto Alegre: Artmed, 2014.

ARIÈS, P. História social da criança e da família. Rio de Janeiro: LTC, 1981.

BACHMANN, C. J.; WIJLAARS, L. P.; KALVERDIJK, L. J.; BURCU, M.; GLAESKE, G.; SCHUILING-VENINGA, C. C. M.; HOFFMANN, F.; AAGAARD, L.; ZITO, J. M. Trends in ADHD medication use in children and adolescents in five western countries, 2005-2012.

European Neuropsychopharmacology, v. 27, n. 5, p. 484-493, 2017.

BARROS, D. B. Os usos e sentidos do metilfenidato: experiências entre o tratamento e o aprimoramento da atenção. Orientador: Francisco Javier Guerrero Ortega. 2014. 182 f. Tese (Doutorado em Saúde Coletiva) - Programa de Pós-Graduação em Saúde Coletiva, Universidade do Estado do Rio de Janeiro, Rio de Janeiro, 2014.

BRZOZOWSKI, F. S.; CAPONI, S. Da encefalite letárgica ao Transtorno de Déficit de Atenção com Hiperatividade (TDAH): emergência e consolidação das explicações biológicas reducionistas. Gavagai, v. 2, n. 2, p. 26-39, 2014.

BRZOZOWSKI, F. S.; CAPONI, S. Medicamentos estimulantes: uso e explicaçóes em casos de crianças desatentas e hiperativas. Cadernos Brasileiros de Saúde Mental, v. 7, n. 15, p. 1-23, 2015.

CALIMAN, L. V. A constituição sócio-médica do "fato TDAH". Psicologia \& Sociedade, v. 21, n. 1, p. 135-144, 2009.

CAPONI, S. Uma sala tranquila: neurolépticos para uma biopolítica da indiferença. São Paulo: LiberArs, 2019.

CONRAD, P.; SCHNEIDER, J. W. Deviance and medicalization. Philapelphia: Temple University Press, 1992.

DALE, R. C.; CHURCH, A. J.; SURTEES, R. A. H.; LEES, A. J.; ADCOCK, J. E.; HARDING, B.; NEVILLE, B. G. R.; GIOVANNONI, G. Encephalitis lethargica syndrome: 20 new cases and evidence of basal ganglia autoimmunity. Brain, v. 127, p. 21-33, 2004.

DARDOT, P.; LAVAL, C. A nova razáo do mundo: ensaio sobre a sociedade neoliberal. São Paulo: Boitempo, 2016.

FINDLING, R. L. Evolution of the treatment of Attention-Deficit/Hyperactivity Disorder in children: a review. Clinical Therapeutics, v. 30, n. 5, p. 942-957, 2008. 
FITZGERALD, M.; BELLGROVE, M.; GILL, M. Handbook of attention deficit hyperactivity disorder. West Sussex: John Wiley \& Sons, Ltd, 2007.

FLYNN, J. R. The mean IQ of Americans: massive gains 1932 to 1978. Psychological Bulletin, v. 95, n. 1, p. 29-51, 1984.

FOUCAULT, M. Vigiar e Punir. Petrópolis: Vozes, 2008.

FOUCAULT, M. Em defesa da sociedade. 2. ed. São Paulo: Martins Fontes, 2010.

FRANCES, A. DSM-5 is a guide, not a bible: simply ignore its 10 worst changes The Huffington Post Science, 2012. Disponível em: http://www.huffingtonpost.com/allen-frances/dsm5_b_2227626.html. Acesso em: 26 jun. 2020.

GOULD, M. S.; WALSH, B. T.; MUNFAKH, J. L.; KLEINMAN, M.; DUAN, N.; OLFSON, M.; GREENHILL, L.; COOPER, T. Sudden death and use of stimulant medications in youths. The American Journal of Psychiatry, v. 166, n. 9, p. 992-1001, 2009.

GUARIDO, R. A biologização da vida e algumas implicaçôes do discurso médico sobre a educação. In: CONSELHO REGIONAL DE PSICOLOGIA DE SÃO PAULO; GRUPO INTERINSTITUCIONAL QUEIXA ESCOLAR (org.). Medicalizaçáo de crianças e adolescentes. São Paulo: Casa do Psicólogo, 2010. p. 29-39.

ILLICH, I. A expropriaçáo da saúde. 3. ed. Rio de Janeiro: Nova Fronteira, 1975.

INTERNATIONAL NARCOTICS CONTROL BOARD (INCB). Psychotropic Substances 2019. Viena: United Nations, 2020.

KANNER, L. The thirty-third Maudsley Lecture: trends in child psychiatry. The Journal of Mental Science, v. 105, n. 440, p. 581-593, 1959.

KORTEKAAS-RIJLAARSDAM, A. F.; LUMAN, M.; SONUGA-BARKE, E.; OOSTERLAAN, J. Does methylphenidate improve academic performance? A systematic review and meta-analysis.

European Child and Adolescent Psychiatry, v. 28, n. 2, p. 155-164, 2019.

LEE, J.; GRIZENKO, N.; BHAT, V.; SENGUPTA, S.; POLOTSKAIA, A.; JOOBER, R. Relation between therapeutic response and side effects induced by methylphenidate as observed by parents and teachers of children with ADHD. BMC Psychiatry, v. 11, n. 70, p. 1-7, 2011.

LIMA, A. C. C.; CAPONI, S. A força-tarefa da psiquiatria do desenvolvimento. Physis: Revista de Saúde Coletiva, v. 21, n. 4, p. 1315-1330, 2011.

MARTINHAGO, F; CAPONI, S. Controvérsias sobre o uso do DSM para diagnósticos de transtornos mentais. Physis: Revista de Saúde Coletiva, v. 29, n. 2, p. 1-19, 2019.

MONCRIEFF, J. The myth of the chemical cure: a critique of psychiatric drug treatment. New York: Palgrave Macmillan, 2008.

MONCRIEFF, J. Magic bullets for mental disorders: The emergence of the concept of an "Antipsychotic" drug. Journal of the History of the Neurosciences, v. 22, p. 30-46, 2013. 
A influência do "modelo centrado na doença" no uso de medicamentos para problemas de aprendizagem na escola | Fabíola Stolf Brzozowski

MTA. The MTA Cooperative Group. A 14-month randomized clinical trial of treatment strategies for attention-deficit/hyperactivity disorder. Archives of General Psychiatry, v. 56, p. 1073-1086, 1999.

PASTURA, G.; MATTOS, P.; ARAÚJO, A. P. de Q. C. Prevalência do Transtorno do Déficit de Atenção e Hiperatividade e suas comorbidades em uma amostra de escolares. Arquivos de Neuropsiquiatria, v. 65, n. 4-A, p. 1078-1083, 2007.

PETERSON, B. S.; POTENZA, M. N.; WANG, Z.; ZHU, H.; MARTIN, A.; MARSH, R.; PLESSEN, K. J.; YU, S. An fMRI study of the effects os psychostimulants on default-mode processing during stroop task performance in youths with ADHD. The American Journal of Psychiatry, v. 166, n. 11, p. 1286-1294, 2009.

RAFALOVICH, A. The conceptual history of attention deficit hyperactivity disorder: idiocy, imbecility, encephalitis and the child deviant, 1877-1929. An Interdisciplinary Journal, v. 22, p. 93-115, 2001.

ROCHA, H. H. P. Educação escolar e higienização da infância. Cadernos CEDES, v. 23, n. 59, p. 39-56, 2003.

ROSS, R. G. Psychotic and manic-like symptoms during stimulant treatment of Attention Deficit Hyperactivity Disorder. The American Journal of Psychiatry, v. 163, n. 7, p. 1149-1152, 2006.

RUTTER, M. Syndromes attributed to "Minimal Brain Dysfunction" in childhood. The American Journal of Psychiatry, v. 139, n. 1, p. 21-33, 1982.

SIGNOR, R. C. F.; BERBERIAN, A. P.; SANTANA, A. P. A medicalização da educação: Implicações para a constituição do sujeito/aprendiz. Educaçáo e Pesquisa, v. 43, n. 3, p. 743-763, 2017.

SINGH, I. Not robots: Children's perspectives on authenticity, moral agency and stimulant drug treatments. Journal of Medical Ethics, v. 39, p. 359-366, 2013.

SINGH, I.; KENDALL, T.; TAYLOR, C.; MEARS, A.; HOLLIS, C.; BATTY, M.; KEENAN, S. Young People's Experience of ADHD and Stimulant Medication: A Qualitative Study for the NICE Guideline. Child and Adolescent Mental Health, v. 15, n. 4, p. 186-192, 2010.

STILL, G. F. [1902] Some abnormal psychical conditions in children: Excerpts from three lectures. Journal of Attention Disorders, v. 10, n. 2, p. 126-136, 2006.

STOREBØ, O. J.; KROGH, H. B.; RAMSTAD, E.; MOREIRA-MAIA, C. R.; HOLMSKOV, M.; SKOOG, M.; NILAUSEN, T. D.; MAGNUSSON, F. L.; ZWI, M.; GILLIES, D.; ROSENDAL, S.; GROTH, C.; RASMUSSEN, K. B.; GAUCI, D.; KIRUBAKARAN, R.; FORSBØL, B.; SIMONSEN, E.; GLUUD, C. Methylphenidate for attention-deficit/hyperactivity disorder in children and adolescents: Cochrane systematic review with meta-analyses and trial sequential analyses of randomised clinical trials. BMJ (Online), v. 351, [s. p.], 2015.

THOMAS, R.; SANDERS, S.; DOUST, J.; BELLER, E.; GLASZIOU, P. Prevalence of attentiondeficit/hyperactivity disorder: A systematic review and meta-analysis. Pediatrics, v. 135, n. 4, p. e994-e1001, 2015. 
TIMIMI, S. Pathological child psychiatry and the medicalization of childhood. New York: Brunner-Routledge, 2002.

VAN DER SCHANS, J.; ÇIÇEK, R.; VARDAR, S.; BOS, J. H. J.; DE VRIES, T. W.; HOEKSTRA, P. J.; HAK, E. Methylphenidate use and school performance among primary school children: A descriptive study. BMC Psychiatry, v. 17, n. 1, p. 1-9, 2017.

WALK, A. The pre-history of child psychiatry. The British Journal of Psychiatry, v. 110, p. 754767, 1964.

Recebido: 27/06/2020

Aceito: 10/08/2020 


\section{The influence of the "disease-centered model" on the use of drugs for learning problems at school}

\section{Abstract}

In this article, I ponder the ways the "disease-centered model" of drug action in Psychiatry, proposed by Joanna Moncrieff, may influence the use of drugs such as methylphenidate (Ritalina ${ }^{\circledast}$ ) for the treatment of Attention Deficit Hyperactivity Disorder (ADHD). Many diagnoses in child mental health are identified at school, for instance ADHD. ADHD is frequently explained as being the result of a brain unbalance, and methylphenidate would be a specific treatment to correct this unbalance. This kind of explanation characterizes the "disease-centered model" of drug action in Psychiatry. We argue that this model amplifies the medicalization of childhood and learning at school, making common the use of drugs to "correct" behaviors and increase academic performance.

Keywords: Medicalization of childhood. Methylphenidate. School. Disease-centered model. 